\title{
Systemic Gauge Theory
}

\author{
Ana Paula Lima* \\ CBPF, Brazil \\ E-mail: ana_crlima@hotmail.com \\ José Chauca \\ CBPF, Brazil \\ E-mail: jlchmecbpf.br \\ Renato Doria \\ AprendaNet, Brazil \\ E-mail: doria@aprendanet.com.br
}

\begin{abstract}
Different topics are bringing the meaning of whole in physics. They are: nature tendency for conglomerates, confinement and complexity. Thus, one should investigate on a whole gauge symmetry. For this, a set of fields transforming under a common gauge parameter is considered. A systemic approach to gauge symmetry is studied. Different fields are introduced in a same abelian group, where their association is given through polynomial transformations. Consequently, new fields strength are introduced under such systemic gauge symmetry. It also introduces the possibility of a mass term without requiring Higgs mechanism. A systemic non-linear abelian Lagrangean is proposed. It is candidate for analysing an electromagnetic systemic phenomena.
\end{abstract}

5th International School on Field Theory and Gravitation April 20-24, 2009

Cuiabá city, Brazil

\footnotetext{
${ }^{*}$ Poster section
} 


\section{INTRODUCTION TO SYSTEMIC SYMMETRY}

Considering the possibility of introducting a set of potential fields $\left\{A_{\mu I}\right\}$ transforming under a symmetry group one proposes a gauge theory based on the whole meaning. Thus, our effort is to interpret the whole meaning in terms of gauge symmetry. This lead us to develop the so-called whole gauge theory. It is a formulation that contains the usual gauge theories and extends it for a region where the parts (quantum numbers) are depending on the whole (set of fields organized by a lagrangean). It brings a step forward in the usual reductionist gauge approach [1].

Under such analysis this work involves just the abelian group. The idea for the whole concept to generate a physical model is by introducting different fields rotating under a same gauge group. It creates a fields-wholeness consisted by fields sharing a common gauge parameter. Then, given a set of fields $\left\{A_{\mu I}\right\}$, the following generic transformation law is proposed:

$$
A_{\mu I}^{\prime}=a_{I K} A_{\mu}^{K}+b_{I L} P^{\prime} L(\alpha) \partial_{\mu} \alpha
$$

where $a_{I K}$ and $b_{I L}$ are coefficients and $P_{I}(\alpha)$ introduces a polynomial expansion in terms of the gauge parameter $\alpha(x), P_{I}(\alpha)=\sum a_{I m} \alpha^{m}$. This expression is an evolution of two cases studied previously which are the linear interconnection $A_{\mu I}^{\prime}=A_{\mu I}+\partial_{\mu} \alpha$ [2] and the polynomial interconnection $A_{\mu I}^{\prime}=A_{\mu I}+P_{I}^{\prime}(\alpha) \partial_{\mu} \alpha$ [3]. Equation (1.1) brings the so-called systemic interconnection, a new systemic gauge invariance where a field can transform itself in a set of another fields [4]. In true, a usual fact, given that it already occurs in non-abelian gauge theories.

Defining as systemic field $A_{\mu I}^{S}=a_{I K} A_{\mu}^{K}$ where the index S means systemic, one gets the antisystemic transformation.

$$
A_{\mu, S}^{I^{\prime}}=A_{\mu}^{I}+a^{I K} b_{K M} P^{M^{\prime}} \partial_{\mu} \alpha
$$

Equations (1.1 and 1.2) are showing relationships between the field and the whole.

Thus, there is a new possibility for introducting the meaning of whole through gauge symmetry. Our purpose here is to build a systemic abelian Lagrangean which provides a gauge invariant set of systemic fields. A possibility for investigating a systemic electromagnetism beyond Maxwell. At cosmological level, probably, nature contains a systemic electromagnetic phenomena. However, the source of this work is just to organize, theoretically, such systemic symmetry.

After the whole principle being a possibility for doing physics through gauge symmetry, a next step is to find out physical motivations. We are going to consider two cases. A first one it to introduce mass without Higgs. A second one is to introduce a new electromagnetism based on light. Basically, it introduces a light physics where light is not more passive as in Maxwell case, but becomes its own source of EM fields. This model provides light with photonic currents and a correspondent photonic charge. This means that there is an electromagnetism beyond the usual electric charge. 


\section{PHYSICAL ENTITIES INVOLVED IN THE SYSTEMIC TRANSFORMATION}

The field strength tensors can be defined and built through the set of fields $\left\{A_{\mu I}\right\}$. In this work, we considered two possibilities. The first consist in a conglomerate of fields: $a_{I} A_{\mu}^{I^{\prime}}, a_{I J} A_{\mu}^{I^{\prime}} A_{v}^{J^{\prime}}$ and $a_{I J K} A_{\mu}^{I^{\prime}} A_{v}^{J^{\prime}} A_{\rho}^{K^{\prime}}$. The second possibility is to establish the antisymmetric and symmetric tensors. The gauge invariance conditions are investigated and the result is shown in the next tables.

\begin{tabular}{||l|l||}
\hline Conglomerate of fields & \multicolumn{1}{c||}{ Invariance Conditions } \\
\hline$a_{I} A_{\mu}^{I^{\prime}}$ & $a_{I} b^{I L} P_{L}^{\prime}=0$ \\
\hline$a_{I J} A_{\mu}^{I^{\prime}} A_{v}^{J^{\prime}}$ & $a_{I J} b^{I L} P_{L}^{\prime}=0 ; a_{I J} b^{J L} P_{L}^{\prime}=0$ \\
\hline$a_{I J K} A_{\mu}^{I^{\prime}} A_{v}^{J^{\prime}} A_{\rho}^{K^{\prime}}$ & $a_{I J K} b^{I L} P_{L}^{\prime}=0 ; a_{I J K} b^{J L} P_{L}^{\prime}=0 ; a_{I J K} b^{K L} P_{L}^{\prime}=0$ \\
\hline
\end{tabular}

Table 1: Gauge invariance conditions for the conglomerate of fields

Defining as field strength the expressions:

$$
\begin{gathered}
F_{\mu v}^{I I^{\prime}}=\kappa^{I} \partial_{v} A_{\mu}^{J^{\prime}}-\kappa^{J} \partial_{\mu} A_{v}^{I^{\prime}} \\
S_{\mu v}^{I J^{\prime}}=l^{I} \partial_{\mu} A_{v}^{J^{\prime}}+l^{J} \partial_{v} A_{\mu}^{I^{\prime}}
\end{gathered}
$$

one gets:

$$
\begin{aligned}
F_{\mu \nu}^{I J^{\prime}}= & F_{\mu v, S}^{I J}+P_{L}^{\prime \prime}\left(\kappa^{I} b^{J L}-\kappa^{J} b^{I L}\right) \partial_{\mu} \alpha \partial_{v} \alpha \\
& +P_{L}^{\prime}\left(\kappa^{I} b^{J L}-\kappa^{J} b^{I L}\right) \partial_{\mu} \partial_{\nu} \alpha \\
S_{\mu \nu}^{I I^{\prime}}= & S_{\mu v, S}^{I J}+P_{L}^{\prime \prime}\left(l^{I} b^{J L}+l^{J} b^{I L}\right) \partial_{\nu} \alpha \partial_{\mu} \alpha \\
& +P_{L}^{\prime}\left(l^{I} b^{J L}+l^{J} b^{I L}\right) \partial_{\nu} \partial_{\mu} \alpha
\end{aligned}
$$

which yields the following gauge invariance conditions:

\begin{tabular}{||l|l||}
\hline Tensors & \multicolumn{1}{c||}{ Invariance Conditions } \\
\hline$F_{\mu \nu}^{I I}$ & Gauge Invariant \\
\hline$S_{\mu \nu}^{I I}$ & $b^{I L} P_{L}^{\prime}=0 ; b^{I L} P_{L}^{\prime \prime}=0 ; \operatorname{det} B=0$ \\
\hline$F_{\mu v}^{I J}$ & $\left(\kappa^{J} b^{I L}-\kappa^{I} b^{J L}\right) P_{L}^{\prime}=0 ;\left(\kappa^{J} b^{I L}-\kappa^{I} b^{J L}\right) P_{L}^{\prime \prime}=0 ; \operatorname{det}\left(\kappa^{M} b^{N M}-\kappa^{N} b^{M N}\right)=0$ \\
\hline$a_{I J} F_{\mu v}^{I J}$ & $a_{[I J]} \kappa^{I} b^{J L} P_{L}^{\prime}=0$ \\
\hline$S_{\mu \nu}^{I J}$ & $\left(l^{J} b^{I L}+l^{I} b^{J L}\right) P_{L}^{\prime}=0 ;\left(l^{J} b^{I L}+l^{I} b^{J L}\right) P_{L}^{\prime \prime}=0 ; \operatorname{det}\left(l^{M} b^{N M}+l^{N} b^{M N}\right)=0$ \\
\hline$b_{I J} S_{\mu v}^{I J}$ & $b_{(I J)} l^{I} b^{J L} P_{L}^{\prime}=0$ \\
\hline
\end{tabular}

Table 2: Gauge invariance conditions for the field strength tensors

Consequently, one notices that the gauge invariance conditions are not necessarily depending of polynomial terms $\left(P_{I}^{\prime}\right)$. This means that the coefficients $a_{I J}, b_{K L}, \kappa_{M}$ and $l_{M}$ can control the gauge invariance. It says that at systemic level one can produce fields conglomerates and symmetric tensors with physical interpretations. 


\section{SGT LAGRANGEAN}

Based on the previous results a Systemic Gauge Theory (SGT) Lagrangean is written as:

$$
\mathscr{L}_{S G T}=\mathscr{L}_{K}+\mathscr{L}_{M}+\mathscr{L}_{I}
$$

Considering the kinetic Lagrangean $\mathscr{L}_{K}$ one gets:

$$
\begin{gathered}
\mathscr{L}_{K}=a_{I J} a_{K L} F_{\mu v}^{I J} F^{\mu v K L}+b_{I J} b_{K L} S_{\mu v}^{I J} S^{\mu v K L}+c_{I J} c_{K L} F_{\mu v}^{I J} S^{\mu v K L} \\
+d_{I J} d_{K L} F_{\alpha}^{\alpha I J} F_{\beta}^{\beta K L}+e_{I J} e_{K L} S_{\alpha}^{\alpha I J} S_{\beta}^{\beta K L}+f_{I J} f_{K L} F_{\alpha}^{\alpha I J} S_{\beta}^{\beta K L}
\end{gathered}
$$

In order to prove its gauge invariance, we are going to consider the following redefinitions:

$$
\begin{gathered}
F_{\mu v}^{I I^{\prime}}=a^{I K} F_{\mu v K K} \equiv F_{\mu v}^{I, S} \\
S_{\mu v}^{I I I^{\prime}}=S_{\mu v}^{I, S}+2 b^{I L} P_{L}^{\prime} \partial_{\mu} \partial_{v} \alpha+2 b^{I L} P_{L}^{\prime \prime} \partial_{\mu} \alpha \partial_{v} \alpha
\end{gathered}
$$

and

$$
\begin{gathered}
a_{(I J)} F_{\mu v}^{I J}=a_{(I J)} \kappa^{I} F_{\mu v}^{J} \\
a_{[I J]} F_{\mu v}^{I J}=a_{[I J]} \kappa^{I} S_{\mu v}^{J} \\
b_{(I J)} S_{\mu v}^{I J}=b_{(I J)} 1^{I} S_{\mu v}^{J} \\
b_{[I J]} S_{\mu v}^{I J}=b_{[I J]} 1^{I} F_{\mu v}^{J}
\end{gathered}
$$
$\left(\hat{\mathscr{L}}_{K}\right)$ :

which yields that the equation (3.2) contains a diagonal sector $\left(\mathscr{L}_{K}\right)$ plus entangled sector

$$
\begin{gathered}
\mathscr{L}_{K}=\mathscr{L}_{K}(F F)+\mathscr{L}_{K}(S S)+\mathscr{L}_{K}(F S)+\hat{\mathscr{L}}_{K}(F F)+\hat{\mathscr{L}}_{K}(S S)+\hat{\mathscr{L}}_{K}(F S) \\
\mathscr{L}_{K}(F F)=a_{(I J)} a_{(K L)} \kappa_{I} \kappa_{K} F_{\mu v}^{J J} F^{\mu v L L}+b_{[I J]} b_{[K L]} l_{I} l_{K} F_{\mu v}^{J J} F^{\mu v L L} \\
+c_{(I J)} c_{[K L]} \kappa_{I} l_{K} F_{\mu v}^{J J} F^{\mu v L L} \\
\mathscr{L}_{K}(S S)=a_{[I J]} a_{[K L]} \kappa_{I} \kappa_{K} S_{\mu v}^{J J} S^{\mu v L L}+b_{(I J)} b_{(K L)} l_{I} l_{K} S_{\mu v}^{J J} S^{\mu v L L} \\
+c_{[I J]} c_{(K L)} \kappa_{I} l_{K} S_{\mu v}^{J J} S^{\mu v L L} \\
\mathscr{L}_{K}(F S)=a_{(I J)} a_{[K L]} \kappa_{I} \kappa_{K} F_{\mu v}^{J J} S^{\mu \nu L L L}+a_{[I J]} a_{(K L)} \kappa_{I} \kappa_{K} S_{\mu v}^{J J} F^{\mu v L L} \\
+b_{(I J)} b_{[K L]} l_{I} l_{K} S_{\mu v}^{J J} F^{\mu v L L}+b_{[I J]} b_{(K L)} l_{I} l_{K} F_{\mu v}^{J J} S^{\mu v L L} \\
+c_{(I J)} c_{(K L)} \kappa_{K} l_{l} F_{\mu v}^{J J} S^{\mu v L L}+c_{[I J]} c_{[K L]} \kappa_{I} l_{K} S_{\mu v}^{J J} F^{\mu v L L}
\end{gathered}
$$


where the term $\mathscr{L}_{K}(F F)$ is gauge invariant and verifying the symmetry properties, the term $\mathscr{L}_{K}(F S)$ is null. The gauge invariance conditions for the terms $\mathscr{L}_{K}(S S), \hat{\mathscr{L}}_{K}(F F), \hat{\mathscr{L}}_{K}(S S)$ and $\hat{\mathscr{L}}_{K}(F S)$ are shown in the section 4 where are introduced the expressions:

$$
\begin{gathered}
\varepsilon_{I K}=a_{[I J]} a_{[K L]} \kappa_{J} \kappa_{L}+b_{(I J)} b_{(K L)} l_{J} l_{L}+c_{[I J]} c_{(K L)} \kappa_{J} l_{L} \\
\bar{\varepsilon}_{I K}=4 d_{[I J]} d_{[K L]} \bar{\kappa}_{J} \bar{\kappa}_{L}+4 e_{(I J)} e_{(K L)} \bar{l}_{J} \bar{l}_{L}+4 f_{[I J]} f_{(K L)} \bar{\kappa}_{J} \bar{l}_{L}
\end{gathered}
$$

The mass term is given by:

$$
\mathscr{L}_{M}^{\prime}=m_{I J}^{2} A_{\mu}^{I^{\prime}} A^{\mu J^{\prime}}
$$

or

$$
\mathscr{L}_{M}^{\prime}=m_{I J}^{2}\left(A_{\mu S}^{I} A^{\mu J S}+b^{J L} P_{L}^{\prime} A_{\mu S}^{I} \partial^{\mu} \alpha+b^{I L} P_{L}^{\prime} A^{\mu J S} \partial_{\mu} \alpha+b^{I L} b^{J L} P_{L}^{\prime} P_{L}^{\prime} \partial_{\mu} \alpha \partial^{\mu} \alpha\right)
$$

Using the definition $A_{\mu S}^{I}=a^{I K} A_{\mu K}, \mathscr{L}_{M}^{\prime}$ must satisfy the following invariance conditions:

$$
\begin{gathered}
m_{I J}^{2}\left(a^{I K} b^{J L}+a^{J K} b^{I L}\right) P_{L}^{\prime} A_{\mu K} \partial^{\mu} \alpha=0 \\
m_{I J}^{2} b^{I L} b^{J L} P_{L}^{\prime} P_{L}^{\prime} \partial_{\mu} \alpha \partial^{\mu} \alpha=0
\end{gathered}
$$

Defining $m_{(I J)}^{2}=m_{I I} m_{J J}$ :

$$
\begin{gathered}
{\left[m_{I I}\left(a^{I K} b^{J L}+a^{J K} b^{I L}\right) P_{L}^{\prime}\right]\left[m_{J J} A_{\mu K}\right]\left[\partial^{\mu} \alpha\right]=0} \\
{\left[m_{I I} b^{I L} P_{L}^{\prime}\right]\left[m_{J J} b^{J L} P_{L}^{\prime}\right]\left[\partial_{\mu} \alpha \partial^{\mu} \alpha\right]=0} \\
{\left[m_{I I} b^{I L}\right]=0} \\
{\left[m_{J J} b^{I L}\right]=0}
\end{gathered}
$$

The equations (3.16) and (3.17) brings the solution to introduce mass without Higgs.

Therefore, appears an alternative mechanism to generate mass diverse from Stueckelberg and Higgs mechanism [5]. Coefficients $m_{I J}, a_{K L}$ and $b_{M N}$ are able to preserve the massive model gauge invariance. The introduction of a whole symmetry allows a kind of symmetry managing. In a further work the interaction terms will be explored [4].

\section{MANAGING SYMMETRY}

In this section, the conditions to manage the symmetry are shown. The next table describes the gauge invariance conditions for the SGT Lagrangean. 


\begin{tabular}{||l|l||}
\hline TGS Lagrangean & \multicolumn{1}{|c||}{ Invariance Conditions } \\
\hline $\mathscr{L}_{M}$ & $\left(b^{L I}\right)^{t} m_{I J}^{2} b^{J L} P_{L}^{\prime} P_{L}^{\prime}=0$ \\
\hline $\mathscr{L}_{K}(S S)$ & $\varepsilon_{I K} S_{\mu v}^{I S} b^{K L} P_{L}^{\prime} \partial^{\mu} \partial^{v} \alpha=0$ \\
& $\varepsilon_{I K} S_{\mu v}^{I S} b^{K L} P_{L}^{\prime \prime} \partial^{\mu} \alpha \partial^{v} \alpha=0$ \\
& $\varepsilon_{I K} b^{I L} S^{\mu}{ }^{K, S} P_{L}^{\prime} \partial_{\mu} \partial_{v} \alpha=0$ \\
& $\varepsilon_{I K} b^{I L} b^{K L} P_{L}^{\prime} P_{L}^{\prime} \partial_{\mu} \partial_{\nu} \alpha \partial^{\mu} \partial^{v} \alpha=0$ \\
& $\varepsilon_{I K} b^{I L} b^{K L} P_{L}^{\prime} P_{L}^{\prime \prime} \partial_{\mu} \partial_{v} \alpha \partial^{\mu} \alpha \partial^{v} \alpha=0$ \\
& $\varepsilon_{I K} b^{I L} S^{\mu}{ }^{K} K P_{L}^{\prime \prime} \partial_{\mu} \alpha \partial_{\nu} \alpha=0$ \\
& $\varepsilon_{I K} b^{I L} b^{K L} P_{L}^{\prime} P_{L}^{\prime \prime} \partial^{\mu} \partial^{v} \alpha \partial_{\mu} \alpha \partial_{\nu} \alpha=0$ \\
& $\varepsilon_{I K} b^{I L} b^{K L} P_{L}^{\prime \prime} P_{L}^{\prime \prime} \partial_{\mu} \alpha \partial_{v} \alpha \partial^{\mu} \alpha \partial^{v} \alpha=0$ \\
\hline \multirow{5}{*}{$\hat{\mathscr{L}}_{K}$} & $\bar{\varepsilon}_{I K} a^{I K} b^{K L} P_{L}^{\prime} \partial_{\alpha} A_{K}^{\alpha} \partial_{\beta} \partial^{\beta} \alpha=0$ \\
& $\bar{\varepsilon}_{I K} a^{I K} b^{K L} P_{L}^{\prime \prime} \partial_{\alpha} A_{K}^{\alpha} \partial_{\beta} \alpha \partial^{\beta} \alpha=0$ \\
& $\bar{\varepsilon}_{I K} a^{K M} b^{I L} P_{L}^{\prime} \partial_{\alpha} \partial^{\alpha} \alpha \partial_{\beta} A_{M}^{\beta}=0$ \\
& $\bar{\varepsilon}_{I K} b^{I L} b^{K L} P_{L}^{\prime} P_{L}^{\prime} \partial_{\alpha} \partial^{\alpha} \alpha \partial_{\beta} \partial^{\beta} \alpha=0$ \\
& $\bar{\varepsilon}_{I K} b^{I L} b^{K L} P_{L}^{\prime} P_{L}^{\prime \prime} \partial_{\alpha} \partial^{\alpha} \alpha \partial_{\beta} \alpha \partial^{\beta} \alpha=0$ \\
& $\bar{\varepsilon}_{I K} a^{K M} b^{I L} P_{L}^{\prime \prime} \partial_{\alpha} \alpha \partial^{\alpha} \alpha \partial_{\beta} A_{M}^{\beta}=0$ \\
& $\bar{\varepsilon}_{I K} b^{I L} b^{K L} P_{L}^{\prime} P_{L}^{\prime \prime} \partial_{\alpha} \alpha \partial^{\alpha} \alpha \partial_{\beta} \partial^{\beta} \alpha=0$ \\
& $\bar{\varepsilon}_{I K} b^{I L} b^{K L} P_{L}^{\prime \prime} P_{L}^{\prime \prime} \partial_{\alpha} \alpha \partial^{\alpha} \alpha \partial_{\beta} \alpha \partial^{\beta} \alpha=0$ \\
\hline
\end{tabular}

Table 3: Managing Symmetry

\section{CONCLUSION}

It is presented a systemic symmetry which proposal is to analyse the nature systemic phenomena. The SGT confirms the concept of whole and works with the systemic gauge transformation. It says that a field can transform itself in a set of another fields. A systemic electromagnetism becomes possible. It can be of direct importance for the interpretation on collective electromagnetic radiations and cosmic magnetism.

\section{References}

[1] 50 years of Yang-Mills, edited by Gerardus't Hooft, World Scientific 2005.

[2] Doria, R. M.; Helayël-Neto, J.A.; Dulinickova, A. Z., Static Potentials From An Extended Gauge Symmetry IL NUOVO CIMENTO, v. 94A, p. 51, 1986.

[3] R.Doria, Whole Gauge Theory, XXX Encontro Nacional de Física de Partículas e Campos 2009. J.Chauca, Doctor degree thesis, in preparation.

[4] Ana Paula Lima and R. Doria, Systemic Gauge Theory, in preparation.

[5] E. C. G. Stueckelberg, Radiation theory for photons of arbitrarily small mass, Helv. Phys. Acta 30, 209-215.

Peter W. Higgs, Symmetries and the Masses of Gauge Bosons, Phys. Rev. Lett. 13, 508. 